\title{
Comparison of experimental and numerical sloshing loads in partially filled tanks
}

\author{
S. Brizzolara, L. Savio \& M. Viviani
}

Y. Chen \& P. Temarel

N. Couty \& S. Hoflack

L. Diebold \& N. Moirod

A. Souto Iglesias

\begin{abstract}
Sloshing phenomenon consists in the movement of liquids inside partially filled tanks, which generates dynamic loads on the tank structure. Resulting impact pressures are of great importance in assessing structural strength, and their correct evaluation still represents a challenge for the designer due to the high nonlinearities involved, with complex free surface deformations, violent impact phenomena and influence of air trapping. In the present paper a set of two-dimensional cases for which experimental results are available are considered to assess merits and shortcomings of different numerical methods for sloshing evaluation, namely two commercial RANS solvers (FLOW-3D and LS-DYNA), and two own developed methods (Smoothed Particle Hydrodynamics and RANS). Impact pressures at different critical locations and global moment induced by water motion for a partially filled tank with rectangular section having a rolling motion have been evaluated and results are compared with experiments.
\end{abstract}

\section{INTRODUCTION}

The sloshing phenomenon is a highly nonlinear movement of liquids inside partially filled tanks with oscillatory motions. This liquid movement generates dynamic loads on the tank structure and thus becomes a problem of relative importance in the design of marine structures in general and an especially important problem in some particular cases (Tveitnes et al. 2004).

In some cases, this water movement is used for dampening ship motions (passive anti-roll tanks), especially for vessels with low service speed (fishing vessels, supply vessels, oceanographic and research ships, etc.) and for which active fin stabilizers would not produce a significant effect (Lloyd 1989).

The sloshing problem has been to a great extent investigated in the last 50 years, with increasing levels of accuracy and computational efforts.
First attempts were based on mechanical models of the phenomenon by adjusting terms in the harmonic equation of motion (Graham \& Rodriguez 1952 , Lewison 1976). These types of techniques are used when time-efficient and not very accurate results are needed (Aliabadi et al. 2003).

The second series of investigations solves a potential flow problem with a very sophisticated treatment of the free-surface boundary conditions (Faltinsen et al. 2005) that extends the classical linear wave theory by performing a multimodal analysis of the free-surface behavior. This approach is very time efficient and accurate for specific applications but it does not allow to model overturning waves and may present problems when generic geometries and/or baffled tanks are considered.

The third group of methods solves the nonlinear shallow water equations (Stoker 1957) with the use of different techniques (Lee et al. 2002, Verhagen \& Van Wijngaarden 1965). 
The fourth group of techniques used to deal with highly nonlinear free-surface problems is aimed at solving numerically the incompressible Navier - Stokes equations. Frandsen (2004) solves the nonlinear potential flow problem with a finite difference method in a 2-D tank that is subjected to horizontal and vertical motion, with very good results, but this approach suffers from similar shortcomings to the multimodal method. Celebi and Akyildiz (2002) solve the complete problem by using a finite difference scheme and a VOF formulation for tracking the free-surface. Sames et al. (2002) present results carried out with a commercial finite volume VOF method applied to both rectangular and cylindrical tanks. Schellin et al. (2007) present coupled ship/sloshing motions with very promising results.

In general, numerical techniques present significant problems when considering highly nonlinear waves and/or overturning waves, the effect of air cushions and fluid-structure interactions. Considering the first problem, Smoothed Particle Hydrodynamics (SPH) meshless method appears as a promising alternative to standard grid based techniques because of their intrinsic capability to capture surface deformations. Literature about SPH applications to typical marine problems is not very abundant; in Colagrossi et al. 2003 one of the first applications is shown. In successive years, a certain number of applications devoted to the assessment of slamming phenomenon (Oger et al. 2006, Viviani et al. 2007a, b 2008) are found. Sloshing phenomenon is considered by Souto Iglesias et al. 2006 and Delorme et al. 2008b, in which a comprehensive series of calculations is performed, focusing attention on resulting global moment and its dependence with tank oscillating frequency, but problems related to the evaluation of local impact pressures are still considerable, with presence of significantly oscillating results.

The activity described in the present paper, which was carried out in the framework of the EU funded MARSTRUCT Network of Excellence, covered three two-dimensional (or infinite length) cases, focusing on impact pressures and global moments into a partially filled tank with rectangular section, which has an oscillatory rolling motion with different periods and different water levels. For these tests, experimental measurements were carried out by the Model Basin Research Group (CEHINAV) of the Naval Architecutre Department (ETSIN) of the Technical University of Madrid (UPM), in the context of a comprehensive analysis of sloshing phenomenon, as reported by Delorme et al. (2007 and 2008a).

A series of numerical techniques has been applied by various participants to assess their merits and shortcomings, and in particular:

- a RANS code own-developed by UoS (University of Southampton)
- two available commercial software for the solution of RANS equations, namely FLOW-3D applied by BV (Bureau Veritas) and LS-DYNA applied by PRI (Principia)

- a SPH code own developed by DINAV (University of Genoa).

\section{EXPERIMENTAL SET-UP}

The experimental tests which are used for benchmarking the various numerical techniques were performed by CEHINAV-ETSIN-UPM, as reported in Delorme et al. 2007 and 2008a.

In particular, a rectangular tank having dimensions (in centimeters) reported in Fig. 1 was considered. The tank is cylindrical, and the dimension perpendicular to those reported in Fig. 1 is $62 \mathrm{~mm}$; a sinusoidal rolling motion has been imposed during experiments, with a rolling axis located $18.4 \mathrm{~cm}$ over the bottom line, an amplitude of $4^{\circ}$ in all cases and different periods.

The tank was fitted for a series of sensors in different locations, as indicated in Fig. 1. During experiments, pressures in correspondence to the two most critical positions were recorded. The sensors are BTE6000 Flush Mount, with a $500 \mathrm{mbar}$ range.

In parallel to pressure measurements, global torque measurement were conducted. In particular, torque time history was measured during experiments with water inside the tank and with empty tank, then the first harmonic of the moment response of the liquid with respect to the tank rotating centre for every case was obtained postprocessing data.

In table 1, the two water levels considered in present analysis are reported, together with the correspondent natural period of oscillation.

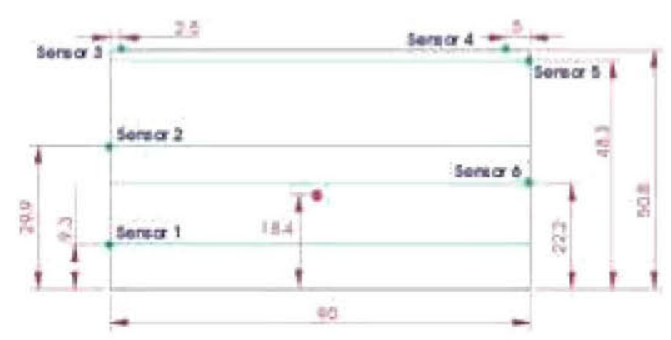

Figure 1. Tank geometry and position of the sensors.

Table 1. Water levels considered.

\begin{tabular}{lcll}
\hline $\begin{array}{l}\text { Level } \\
\text { symbol }\end{array}$ & Level $[\mathrm{cm}]$ & $\begin{array}{l}\text { Level/ } \\
\text { tank height }\end{array}$ & $\begin{array}{l}\text { Natural } \\
\text { Period } \mathrm{T}_{0}[\mathrm{~s}]\end{array}$ \\
\hline $\mathrm{A}$ & 9.3 & $18.3 \%$ & 1.91 \\
$\mathrm{~B}$ & 22.2 & $43.7 \%$ & 1.32 \\
\hline
\end{tabular}


Table 2. Test Cases description.

\begin{tabular}{llll}
\hline Case & Level/tank height & Period $[\mathrm{s}]$ & Period/T \\
\hline 1 & $\mathrm{~A}-18.3 \%$ & 1.91 & 1.0 \\
2 & $\mathrm{~B}-43.7 \%$ & 1.19 & 0.9 \\
3 & $\mathrm{~B}-43.7 \%$ & 1.32 & 1.0 \\
\hline
\end{tabular}

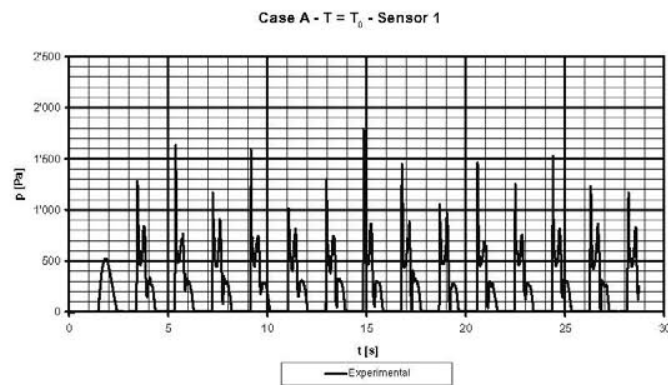

Figure 2. Test Case $1-$ Level $\mathrm{A}-\mathrm{T}=\mathrm{T}_{0}-$ Pressure at Sensor 1.

For both water levels, experiments carried out in correspondence to the resonance period have been considered (case 1 and 3 for level $\mathrm{A}$ and $\mathrm{B}$ respectively), to have large free surface deformation and analyse codes' capability to capture it. Moreover, for case B a lower oscillating period $(90 \%$ of the resonance one) has been analysed (case 2 ), in which a marked beating phenomenon has been observed.

In table 2, main characteristics of cases analysed are briefly summarized for a better understanding.

In correspondence to case 1 , pressure sensors are located at positions 1 and 6 , and impacts were recorded on sides in correspondence to lower sensor, as presented in following Fig. 2.

In correspondence to cases 2 and 3, pressure sensors were located at positions 3 and 6; as anticipated, case 2 is interesting for the presence of beating type kinematics and pressures, with peak values oscillating, as presented in following Fig. 3 for sensor 6 . Regarding case 3 , pressure peaks were recorded in correspondence to both locations as reported in Fig. 4, with impact events at the tank top (sensor 3 ) and pressure rises due to the incoming wave and to water fall after impact at sensor 6 .

In addition to pressure measurement, in following table 3 the resulting first harmonic of the liquid moment is reported.

In particular, $\mathrm{M}_{0}$ is the first harmonic amplitude and $\phi$ is the phase lag with respect to oscillating motion, resulting in equation (1) for time history, considering the motion period $\mathrm{T}$ :

$M=M_{0} \sin \left(\frac{2 \pi}{T} t-\phi\right)$

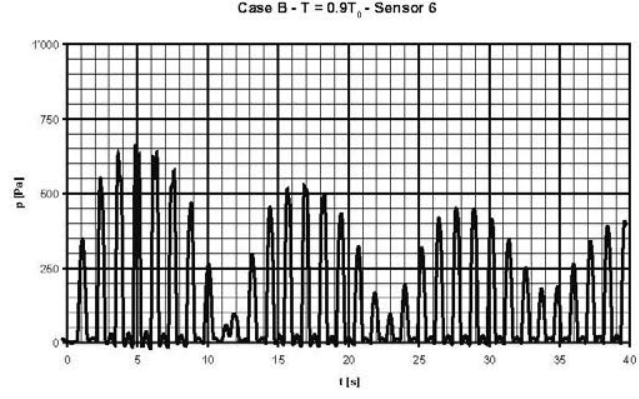

Figure 3. Test Case 2-Level B-T $=0.9 \mathrm{~T}_{0}$-Pressure at Sensor 6 .

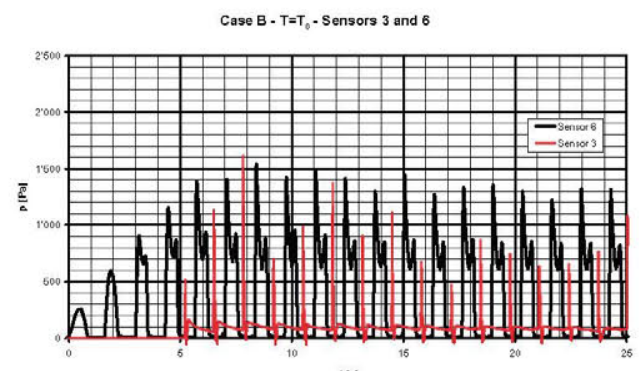

1 s:

Figure 4. Test Case 3-Level $\mathrm{B}-\mathrm{T}=\mathrm{T}_{0}$-Pressure at Sensors 36 .

Table 3. Oscillating moment first harmonic values.

\begin{tabular}{llcr}
\hline Case & $\mathrm{M}_{0}[\mathrm{Nm}]$ & $\mathrm{M}_{0}[\mathrm{Nm} / \mathrm{m}]$ & $\phi[\mathrm{deg}]$ \\
\hline 1 & 4.52 & 72.87 & 84.4 \\
2 & 1.07 & 17.22 & 173.6 \\
3 & 6.70 & 108.03 & 112.0 \\
\hline
\end{tabular}

First harmonic $\mathrm{M}_{0}$ value is reported both for the real experimental case (tank with $62 \mathrm{~mm}$ third dimension) and for the equivalent two-dimensional configuration, for which moment per unit length is given.

Rolling motion during experiments was a pure sinusoidal motion apart at the very beginning of the experiment to avoid infinite accelerations.

Finally, a proper uncertainty analysis has not been conducted yet since it is hard to find a consistent approach to it in this case. This is due to the strong chaotic character of the pressure peaks, as discussed in Delorme et al. 2008, where the initial steps to such analysis were given.

\section{DESCRIPTION OF METHODS}

Methods used for modeling the sloshing phenomenon are summarized in Tables 4 and 5 for test case 1 and 
Table 4. Details of method and idealization-Test case 1.

\begin{tabular}{lll}
\hline Participant & Method & Idealisation details \\
\hline UoS & RANS & $55 \times 50$ grid \\
DINAV & SPH & 37138 real particles \\
& & 1876 virtual particles \\
PRI & LS-DYNA & $90 \times 51$ grid \\
BV & FLOW 3D & $5 \times 220 \times 122$ grid \\
\hline
\end{tabular}

Table 5. Details of method and idealization - Test case 2-3.

\begin{tabular}{lll}
\hline Participant & Method & Idealisation details \\
\hline UoS & RANS & $90 \times 50$ grid \\
DINAV & SPH & 88652 real particles \\
& & 1876 virtual particles \\
PRI & LS-DYNA & $90 \times 51$ grid \\
BV & FLOW 3D & $5 \times 90 \times 62$ grid \\
\hline
\end{tabular}

for test cases 2 and 3 , respectively; in the same tables, some details of the idealization are also included.

In SPH real particles are used to represent water, while virtual ones are used to represent the moving tank. For both of them a diameter of $1.5 \mathrm{~mm}$ has been adopted. BV calculations are 3-dimensional, and the experimental setup was effectively reproduced, while in all other cases a two-dimensional problem was analyzed. Only UoS considered air (and pressure variations inside it), while in all other cases only water was considered (in VoF methods, air is schematized as void). Finally, effective time histories were applied both by BV (case 1 only) and PRI, while pure harmonic oscillations were applied by BV (case 2 and 3 ), UoS and DINAV; it resulted from calculations that this has not a significant impact once phenomena are stabilized.

\subsection{RANS approach adopted by UoS}

The level set formulation in a generalized curvilinear coordinates system has been developed to simulate the free surface waves generated by moving bodies or sloshing of fluid in a container. The Reynoldsaveraged Navier-Stokes (RANS) equations are modified to account for variable density and viscosity for the two-fluid flows (i.e. water-air). By computing the flow fields in both the water and air regions, the location and transport of free surface inside a tank is automatically captured. A detailed description of the numerical method can be found in Chen et al. 2008.

In the simulation of sloshing, appropriate boundary conditions need to be imposed to calculate the impact loads. Traditionally, in a single phase flow solver, a thin artificial buffer zone is adopted near the tank ceiling and a linear combination of free surface and rigid
Table 6. Outline of sloshing cases.

\begin{tabular}{lll}
\hline Case & $\varepsilon$ & Time step [s] \\
\hline Case 1 & $1.5 * \Delta \mathrm{z}$ & $1.0 \times 10^{-3}$ \\
Case 2 & $1.5 * \Delta \mathrm{z}$ & $1.0 \times 10^{-3}$ \\
Case 3 & $1.5 * \Delta \mathrm{z}$ & $1.0 \times 10^{-3}$ \\
\hline
\end{tabular}

wall conditions imposed inside the buffer zone. The magnitude of the impact pressure is affected by several factors such as the choice of time step and thickness of the buffer zone, as discussed in Chen et al. 2008. In the present investigation there is no special treatment required for the free surface as a two-fluid approach is used to solve the RANS equations in both water and air regions in a unified manner and the interface is only treated as a shift in fluid properties. The solid wall boundary condition is imposed by vanishing the normal velocity to the wall or setting the components of velocity on the wall to zero. The wall pressure is obtained by projecting the momentum equation along the normal to the wall.

In addition to data already provided in previous paragraph, other computational conditions are listed in Table 6 , indicating the values of half the finite thickness of the interface in which density and viscosity change and time step increment examined in this study ( $\Delta \mathrm{z}$ represents the vertical distance between two grid nodes and $\varepsilon$ the interface half thickness).

Regarding torque calculations, for this method they are provided for case 1 only since some problems have been encountered for other cases, which are still under investigation.

\subsection{LS-DYNA approach adopted by PRI}

The LS-DYNA software is used. This is an explicit finite element code solving the mechanics equations (here corresponding more precisely to the RANSE with Reynolds stresses neglected). The fluid domain is modelled using a multi-materials Eulerian formulation. For the advection of the variables at the integration points, a Van Leer scheme (second order precision) is used and a Half Index Shift (HIS) method for the nodal variables. Each material is characterized by a volume fraction inside each element. It interacts in the calculation of the "composite" pressure for the partially filled cells within a Volume Of Fluid (VOF) method. The dispersion of the volume fraction for these particular cells is limited with an interface reconstruction method (boundary between materials modelled as a plane). Air is modeled as void.

The problem is addressed in two dimensions, although under-integrated 8-noded elements (one layer in the direction normal to the plane) are used 


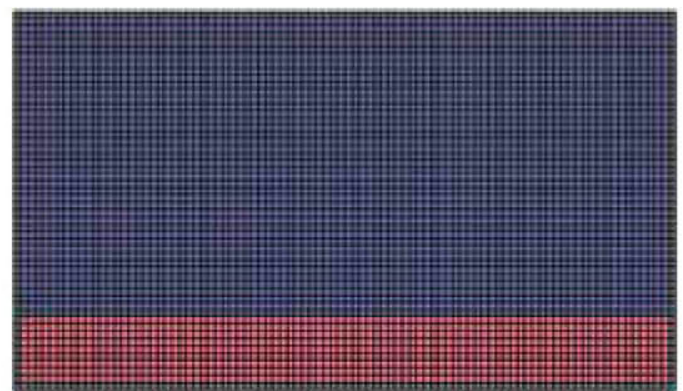

Figure 5. Eulerian mesh for case 1 (water in red, void in blue).

to model the fluid domain in practice. The size of the fluid cells is of $10 \mathrm{~mm}$ edge length, chosen close to the sensor size. The mesh, using $90 \times 51$ solid elements, can be seen in Fig. 5 for cases 1. Cases 2 and 3 are schematized in the same way, except that level of water is modified in accordance to test setup.

The behaviour of water is modelled with a polynomial equation of state, and no viscosity is defined.

The fluid domain is surrounded with one layer of rigid elements (in grey on Fig. 5), modeling the wall and preventing normal flow. Besides, the movement (as given by the roll time histories) is imposed to this rigid part, then the rigid movement of the Eulerian grid (following the walls) is achieved by forcing the grid to follow the movement of three nodes of the rigid part (Aquelet et al. 2003).

The time scheme is based on the Finite Difference method, which is conditionally stable. Thus, the time step is linked to the shortest duration for an acoustic wave to cross any element of the model (fluid or solid), close to $6 \mu$ s here.

Pressure histories are directly post-treated in solid elements where sensors are located.

\subsection{Smoothed particle hydrodynamics method, adopted by DINAV}

SPH is a Lagrangian meshless CFD method, initially developed for compressible fluids (Gingold and Monaghan 1977) and successively adapted and corrected for hydrodynamic problems (Liu and Liu, 2003). The continuum is discretized in a number of particles, each representing a certain finite volume of fluid, which are followed in a Lagrangian way during their motions induced by internal forces between nearby interacting particles and external mass forces or boundary forces. Internal forces derive from the usual Navier-Stokes and continuity equations, made discrete in space by means of a kernel formulation. In general, if $\mathrm{A}$ is a field variable and $\mathrm{W}$ is the kernel function, the following equations (already in their discretised form) are adopted:

$\langle A(r)\rangle=\sum_{j=1}^{N} \frac{m_{j}}{\rho_{j}} A\left(r_{j}\right) W\left(r-r_{j}, h\right)$

where $\mathrm{m}_{j}$ and $\rho_{j}$ are mass and density of the $j$ th particle, $r$ and $r_{j}$ are position vectors in space and for the $j$ th particle, respectively. The term $h$ represents the smoothing length, which determines the extent to which a certain particle has influence on the others. Different kernel functions may be utilized (Liu and Liu 2003); in particular, in the present work, Gaussian kernel was adopted. The resulting formulae, in the particles approximation, for the continuity equation and the momentum equation are:

$$
\begin{aligned}
& \partial \rho_{i} / \partial t=\sum m_{j}\left(v_{i}-v_{j}\right) \cdot \nabla W\left(r-r_{j}, h\right), \\
& \partial v_{i} / \partial t=-\sum m_{j}\left(p_{i} / \rho_{i}^{2}+p_{j} / \rho_{j}^{2}\right) \nabla W\left(r-r_{j}, h\right)+g
\end{aligned}
$$

Moreover, in order to close the problem, an equation of state (Monaghan 1994) which relates density to pressure for each particle, considering the fluid as "weakly compressible", is adopted:

$p=\frac{c_{0}^{2} \rho_{0}}{\gamma}\left[\left(\frac{\rho}{\rho_{0}}\right)^{\gamma}-1\right]$

where $\rho_{0}$ is reference density $\left(1000 \mathrm{~kg} / \mathrm{m}^{3}\right)$ and $\rho$ is density of each particle. Constant $\gamma$ is set to 7 in accordance to Batchelor 1967, providing satisfactory results for various SPH applications. The value of sound speed $c_{0}$ cannot, in general, be set to its effective value for practical reasons (i.e. time steps become too small); thus, it is usually set in order to limit the Mach number to a value below 0.1 (Monaghan 1994) and, consequently, density variations in the incompressible flow to acceptable values. In particular, in accordance to previous works (Viviani et al. 2008), sound speed was set in order to limit density variations to values below $1 \%$; for all cases considered, a value of $50 \mathrm{~m} / \mathrm{s}$ was sufficient to achieve this condition; according to this, a time step of $10^{-5} \mathrm{~s}$ was adopted, which is about half the one required by the simple application of Courant condition (according to previous experience).

Since SPH can be affected by a lack of stability, various authors developed different methods which can help in reducing this problem, such as Artificial Viscosity and XSPH (Monaghan 1992); in present calculations, on the basis of previous experiences (Viviani et al. 2007a, b), it was decided to avoid $\mathrm{XSPH}$, and to consider an artificial viscosity term as reported in equations (5) where $\mathrm{c}, \rho$, and $\mathrm{h}$ are sound 
speed, density, smoothing length and kernel function respectively and the subscript ' $i j$ ' represents a mean value.

$$
\begin{aligned}
& \Pi_{i j}= \begin{cases}\frac{-\alpha c_{i j} \phi_{i j}+\beta \phi_{i j}^{2}}{\bar{\rho}_{i j}} & \vec{v}_{i j} \cdot \vec{x}_{i j}<0 \\
0 & \vec{v}_{i j} \cdot \vec{x}_{i j} \geq 0\end{cases} \\
& \phi_{i j}=\frac{h_{i j} \vec{v}_{i j} \cdot \vec{x}_{i j}}{\left|\vec{x}_{i j}\right|^{2}+\left(\varepsilon h_{i j}\right)^{2}}
\end{aligned}
$$

A comprehensive analysis was carried out to obtain the best setup of parameters $\alpha, \beta$ and $\varepsilon$ (results cannot be included for space limitations), and after it they were all set to the low value of 0.01 , thus almost neglecting also artificial viscosity.

With reference to boundary treatment, repulsive forces are used as suggested by Monaghan (1994), adopting a force which is dependent on the inverse of the distance between fluid and boundary particles according to the formulation of Lennard-Jones for molecular force, as described by Equation (6), where $\mathrm{p}_{1}=12$ and $\mathrm{p}_{2}=6, \mathrm{r}_{0}$ is the cutting-off distance, approximately equal to the smoothing length, and $\mathrm{r}$ is distance between real and boundary particles:

$f(r)=D \frac{r}{r^{2}}\left(\left(\frac{r_{0}}{r}\right)^{p 1}-\left(\frac{r_{0}}{r}\right)^{p 2}\right) \quad$ when $\mathrm{r}<\mathrm{r}_{0}$

$f(r)=0, \quad$ elsewhere

Finally, in order to evaluate pressure an approach similar to the one presented by Oger et al. (2006) was adopted, albeit simplified since pressure is evaluated as a mean of values computed for real particles in proximity of the boundary particle, i.e. in a rectangular region with width parallel to boundary surface and height perpendicular to boundary surface which are multiples of the smoothing length (in particular, a $4 \times 10$ region was considered).

\subsection{Flow-3D approach, adopted by $B V$}

FLOW-3D solves the transient Navier-Stokes equations by a finite volume/finite differences method in a fixed Eulerian rectangular grid. One of its distinctive features is the Fractional Area Volume Obstacle Representation (FAVOR) technique, which allows for the definition of solid boundaries within the Eulerian grid. Using such a technique definition of boundaries and obstacles is carried out independent of the grid generation.

In particular, the 1-phase flow option was used for this calculation; regarding time, the real time roll series was used as input motion for Case A, while for case $\mathrm{B}$ (both $\mathrm{T}=0.9 \mathrm{~T}_{0}$ and $\mathrm{T}=\mathrm{T}_{0}$ ), harmonic excitations were adopted for the sake of simplicity, since it was found that differences were not important. Finally the $\mathrm{x}$-direction was also considered, with a "depth" equal to $6.2 \mathrm{~cm}$, as utilized also in the experiments.

\section{RESULTS AND DISCUSSION}

\subsection{Test case 1-Water Level $A-T=T_{0}$}

In general, this is the case for which the most comprehensive analysis was carried out. In particular, a rather large number of computations was performed with SPH to analyse the effect of some parameters, and in particular of time step and of artificial viscosity; results of this analysis are not included in present paper for space limitations, and only final setup results are presented (time step equal to $10^{-5} \mathrm{~s}, \mathrm{XSPH}$ and artificial viscosity parameters set to 0.01 ). It has to be mentioned, however, that influence of artificial viscosity is very pronounced, and in particular higher values result in an overdamping of all phenomena (with impact pressures almost vanishing in correspondence to values of $\alpha$ and $\beta$ in artificial viscosity equal to 0.3 ), without overturning waves, and with lower pressure peaks.

In Figs. 6 and 7, pressure at Sensor 1 results obtained with all methods are summarized (Fig. 6 represents results obtained in the first part of the simulation/experiment, while Fig. 7 represents a larger

Case A - $\mathrm{T}=\mathrm{T}_{0}$ - Sensor 1

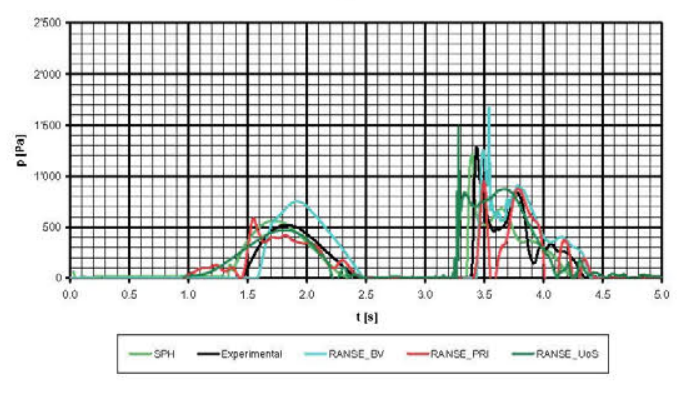

Case $A-T=T_{0}-$ Sensor 1

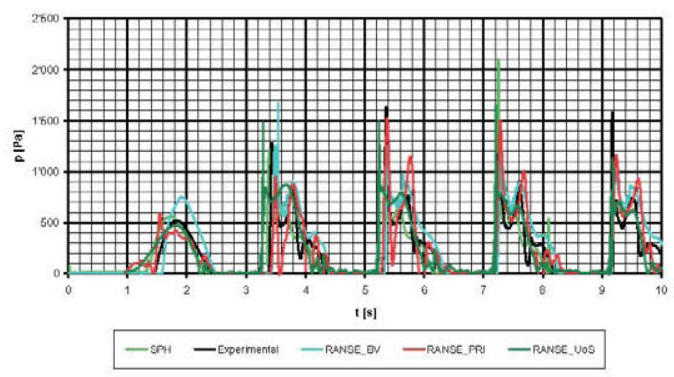

Figure 6. Test Case $1-$ Level $\mathrm{A}-\mathrm{T}=\mathrm{T}_{0}-$ Sensor 1 pressure time history first two oscillations (upper) and up to $\mathrm{t}=10 \mathrm{~s}$ (lower). 
Case A - $T=T_{0}$ - Sensor 1

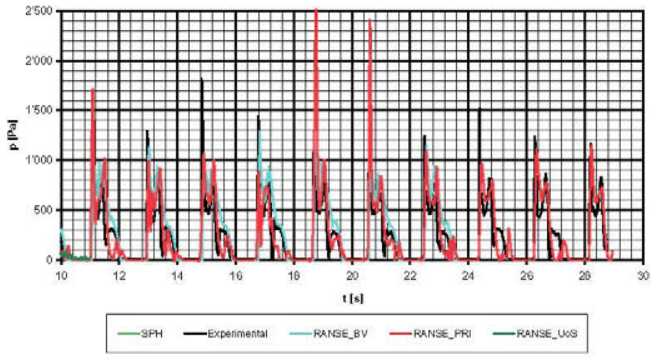

Figure 7. Test Case 1 - Level $\mathrm{A}-\mathrm{T}=\mathrm{T}_{0}-$ Sensor 1 pressure time history for $\mathrm{t}=10-30 \mathrm{~s}$.

scale); in general, pressure peak order of magnitude is captured by all methods applied.

For all of them, first peak is captured correctly (considering mean values in the whole time history), and the second peak is also captured, even if its amplitude accuracy is not as good as the one of the first peak. SPH calculations provide a higher peak at fourth oscillation, similar to some values obtained by PRI at longer time, while UoS results provide a peak which has the correct order of magnitude but a narrower form.

Looking at Fig. 7 for longer times (only BV and PRI calculations have been extended to that), both methods still provide accurate results, even if BV calculations are probably more robust (with lower oscillations) and similar to experimental ones. Calculations with $\mathrm{SPH}$ were not extended to these higher times because of long calculation times, and attention was paid to calibration of various parameters as explained before. Once a satisfactory setup was obtained, this was applied to other cases to better analyze its general nature.

In following Fig. 8 results obtained for sensor 6 are reported. In this case, pressure values are lower by an order of magnitude with respect to those encountered for previous Sensor 1, representing more a "sensor wetting" (thus a less critical point) rather than a real impact phenomenon.

Keeping this in mind, only BV and UoS methods are able to reproduce the experimental pressure peaks, while SPH overestimates them and provides in some cases anomalous negative values (which are present also for UoS); in all cases a time history different from the experimental one is produced, with faster pressure decay than recorded.

PRI method seems only to be capable of signaling the "senor wetting" occurrences after the first $10 \mathrm{sec}-$ onds of calculations, with the presence of a repetitive phenomenon with the same period of the experimental one, however pressure peaks seem to be most of time overestimated, and anomalous negative values result.

In following Fig. 9, results in terms of global torque, as evaluated by DINAV, UOS and PRI are reported.

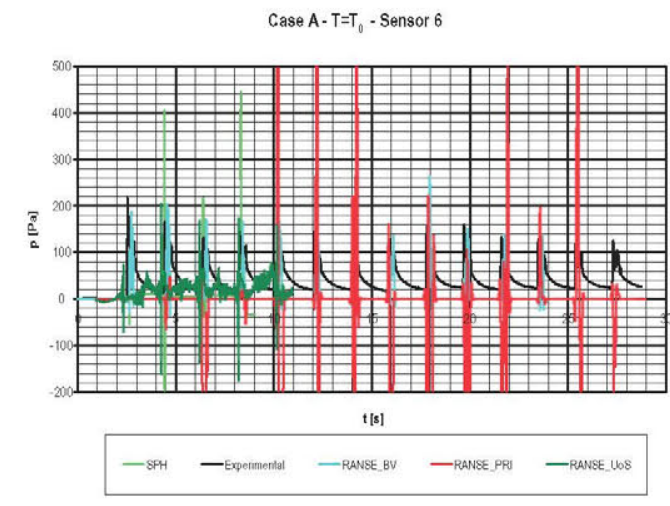

Figure 8. Test Case 1-Level $\mathrm{A}-\mathrm{T}=\mathrm{T}_{0}$-Sensor 6 pressure time history up to $\mathrm{t}=30 \mathrm{~s}$.

Case A. T=T, Torque

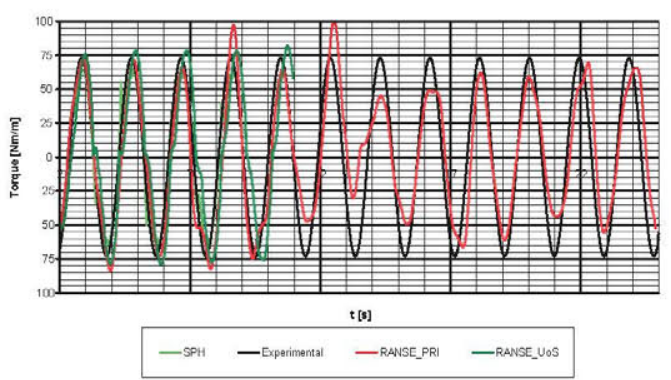

Figure 9. Test Case 1-Level $\mathrm{A}-\mathrm{T}=\mathrm{T}_{0}-$ Global torque time history up to $\mathrm{t}=25 \mathrm{~s}$.

Only results after the first period are reported, in order to look at the "stabilized values" cutting the initial transient phase; this allows a better comparison with experimental results which, as already underlined, are referred only to the first harmonic of oscillating torque time history.

Regarding SPH calculations, results reported are referred as before to the best setup from preliminary analysis. It has to be noticed, however, that once global torque is considered instead of local pressure, differences between various setups are reduced, with the presence of similar results for a wide range of settings of artificial viscosity terms. This result is due to the fact that pressure integration needed to obtain the resultant torque acts like a low-pass filter, smoothing pressure oscillations and cutting the effect of localized pressure peaks. Differences can still be found in correspondence to torque peaks, with values obtained in correspondence to the lowest $\alpha$ and $\beta$ values in artificial viscosity which are about $10-15 \%$ higher than the correspondent ones obtained with high $\alpha$ and $\beta$. 

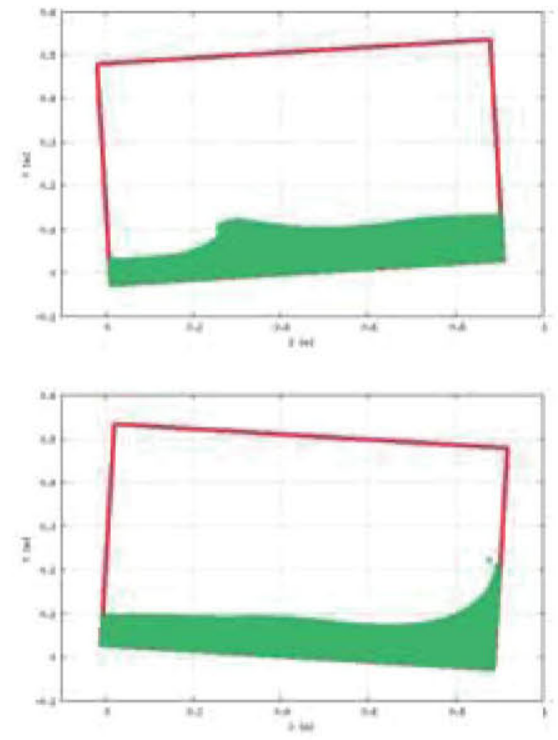

Figure 10-11. Kinematics capturing with $\mathrm{SPH}-$ overturning wave (upper) and flow rise after impact (lower).
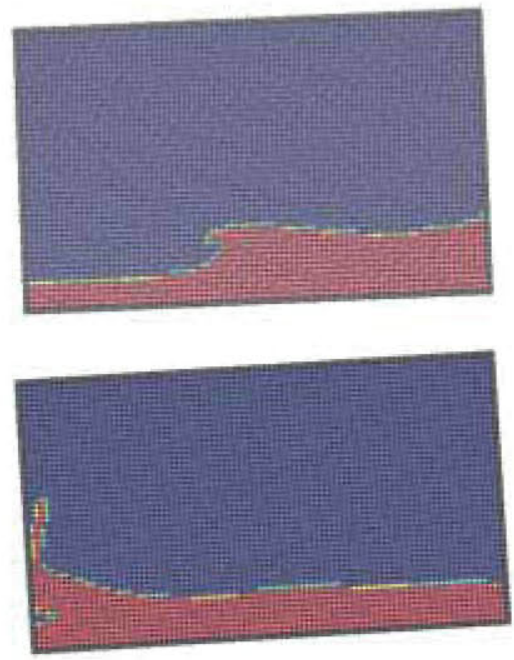

Figure 12-13. Kinematics capturing with LS-DYNAoverturning wave (upper) and flow rise after impact (lower).

Comparing results to experiments, $\mathrm{SPH}$ tends to underestimate by about $10 \%$ the effective oscillating torque amplitude. PRI results are more consistent with experiments, even if in the central part of the simulation they present an anomalous behavior, with a sort of beating phenomenon. UoS results are probably the best with a lower mean error and a higher stability, even if they are limited to the first 10 seconds.
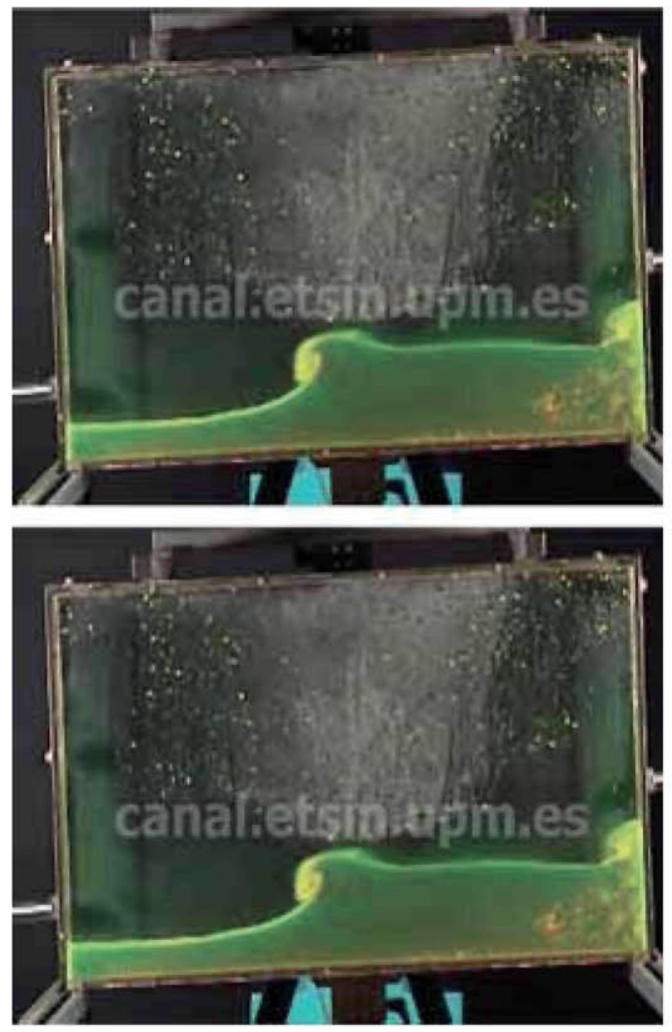

Figure 14-15. Kinematics during experiments-overturning wave at two successive periods.

Finally, in following figures some examples of kinematic capturing are presented (10-11 for SPH, 12-13 for LS-DYNA, 14-15 for experiments), showing a good qualitative capturing of sloshing impact phenomena and of overturning waves.

\subsection{Test case 2 - Water Level B $-T=0.9 T_{0}$}

In Figs. 16-17, results obtained with all methods for Sensor 6 are summarized; as already mentioned, this case is considerably different from the others, since strong impacts are not expected, but just flow oscillations; interest in this case is due to the presence of a beating type phenomena, with pressure peaks of oscillating amplitude and not periodic with the same value for each period.

For all methods applied the beating phenomenon is captured qualitatively, even if most of the numerical codes do not allow to simulate the almost complete quiescence of fluid with pressures near to zero. Only PRI calculations allow obtaining the low pressures in correspondence to the beating, even if they seem 
Case B $-\mathrm{T}=0.9 \mathrm{~T}_{0}-$ Sensor 6

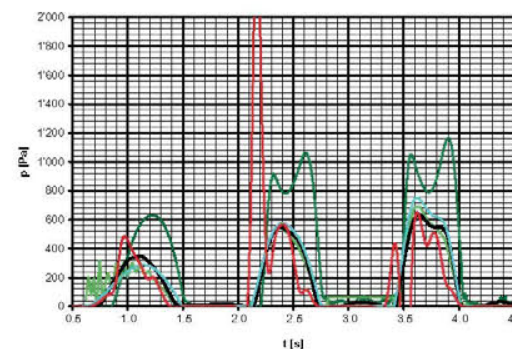

I's]

Case $B-T=0.9 T_{0}-$ Sensor 6

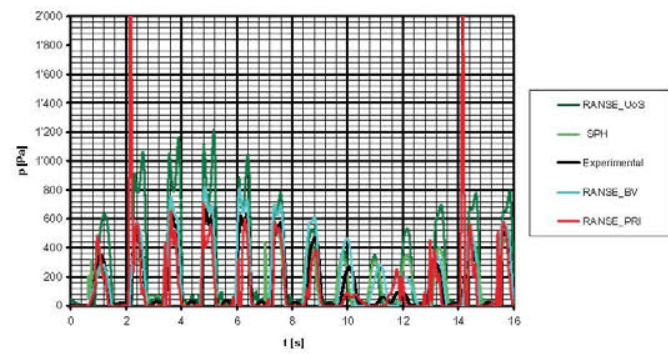

Figure 16. Test Case 2-Level B-T $=0.9 \mathrm{~T}_{0}$ - Sensor 6 pressure time history first three oscillations (upper) and up to $\mathrm{t}=16 \mathrm{~s}$ (lower).

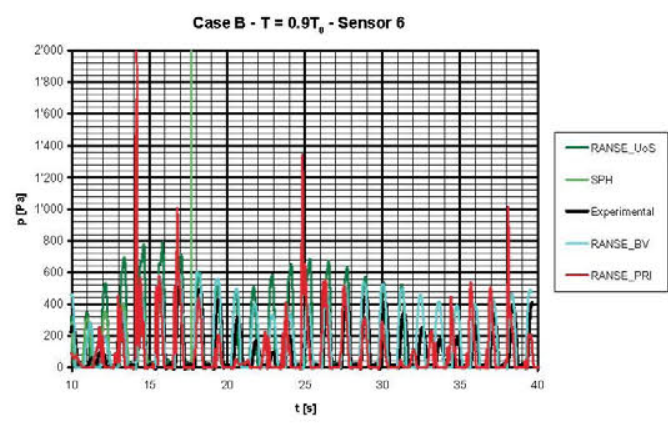

Figure 17. Test Case 2-Level B-T $=0.9 \mathrm{~T}_{0}$-Sensor 6 pressure time history $\mathrm{t}=10-40 \mathrm{~s}$.

to present higher instability than obtained with other methods.

Regarding pressure peaks, UoS calculations overestimate them in the whole time history, while other methods seem to capture them with a higher accuracy; in particular, SPH and PRI capture correctly maximum pressures of the first beating, while BV overestimate them. SPH and BV methods capture pressure curve shapes in the best way, with a sort of secondary peak during "excitation phase" and a more "bell-shaped" curve in the "damping phase"; two anomalous pressure peaks were encountered during calculations by

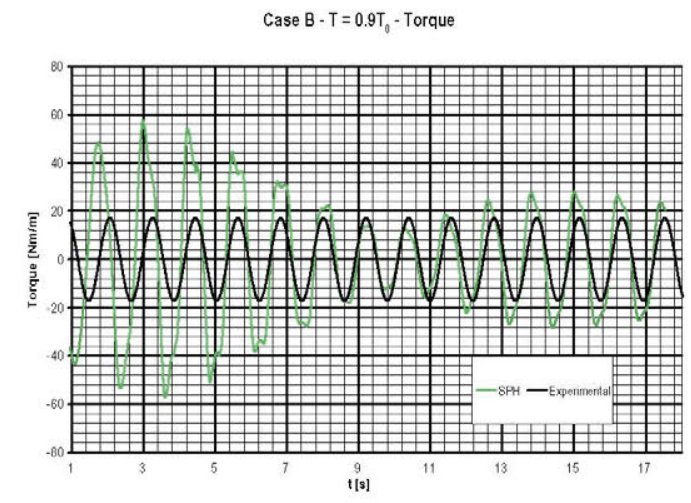

Figure 18. Test Case 2-Level $\mathrm{B}-\mathrm{T}=0.9 \mathrm{~T}_{0}-$ Global torque time history up to $\mathrm{t}=20 \mathrm{~s}$.

means of SPH, showing some instability, while BV calculations seem more robust.

Looking at longer times, PRI calculations continue to capture the beating amplitude (even if with higher peaks in the "excitation phase" and lower peaks in the "damping phase"), while other methods tend to obtain a smoother phenomenon, with lower oscillations due to a possible overdamping and, in general, with overestimated pressures; timing of the beating phenomenon is not correctly captured by PRI and UoS, which show an apparent shift with increasing calculation time, while BV methods captures it correctly. SPH calculations have not been extended to the highest time to avoid too high computational times.

In following Fig. 18, results in terms of global torque, as evaluated with $\mathrm{SPH}$ are reported.

In this case the comparison with experimental results is more difficult than in previous paragraph; this is due to the fact that experimental data are provided in terms of first harmonic of torque oscillations, which in this case of the beating phenomenon does not describe satisfactorily the complete time history. As already mentioned regarding pressure time histories, the method is able to capture the beating phenomenon. Torque values are qualitatively reasonable for the first beating even if, as already noted for pressure results, torque does not vanish in correspondence to the quiescent part. For the second beating results seem to be more damped, consistently with pressure results. Finally, In following Figs. 19-20 and 21-22 some examples of kinematics capturing with SPH and RANS method by PRI, respectively, are reported; in particular, maximum and minimum fluid oscillation during the pseudo-period are shown.

Maximum oscillations are similar for SPH and PRI, while lowest oscillations are not completely captured by SPH, while PRI captures an almost horizontal surface, in accordance with pressure results. 

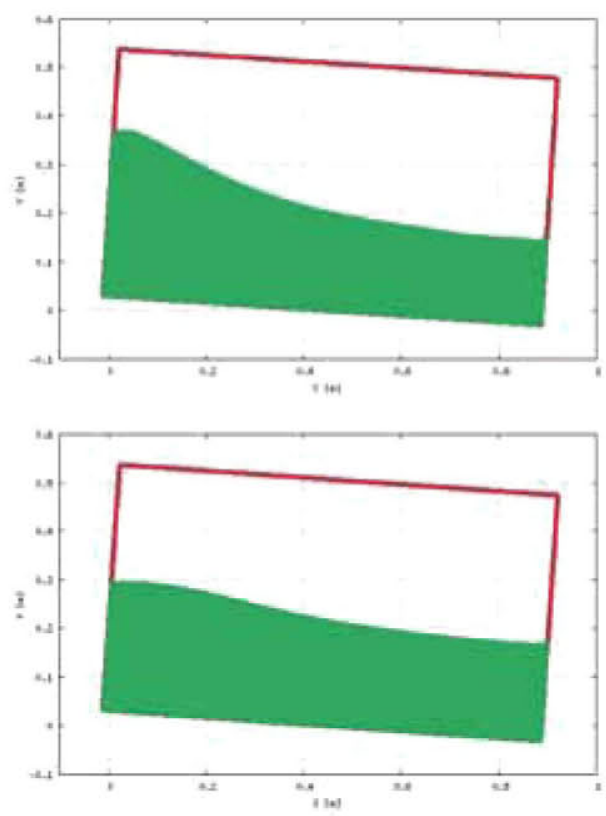

Figure 19-20. Kinematics capturing with SPH-max peak (upper) and min peak (lower).

\subsection{Test case 3-Water Level B-T $=T_{0}$}

While in previous test case very violent impact phenomena were not recorded during experiments, in this case impacts were recorded in correspondence to the pressure gage on the tank top (sensor 3), and pressure peaks were recorded also in correspondence to the pressure gage placed on the tank side in correspondence to the calm water level (sensor 6), despite smoother flow motions are found.

In Figs. 23-24 and 25, results obtained with all methods for Sensor 6 and Sensor 3 respectively are summarized.

In general, pressure peaks order of magnitude for sensor 6 have been captured by all methods applied, while sensor 3 presents higher problems, similarly to what was remarked for sensor 6 in case 1 .

In particular, if Sensor 6 results are considered, mean experimental pressure peak value is about 1300 $\mathrm{Pa}$ and all methods tend to overestimate it, in particular $\mathrm{BV}, \mathrm{SPH}$ and UoS results are about $25-30 \%$ higher, while PRI has a more oscillating behavior, with maximum values about $80 \%$ higher and minimum values $30 \%$ lower than the experimental ones, even if the trend is captured satisfactorily. Regarding pressure time history, $\mathrm{SPH}$ and $\mathrm{BV}$ results seem to be the ones which reproduce better the secondary pressure peak, while UoS presents a larger second peak, and PRI has an intermediate behavior.
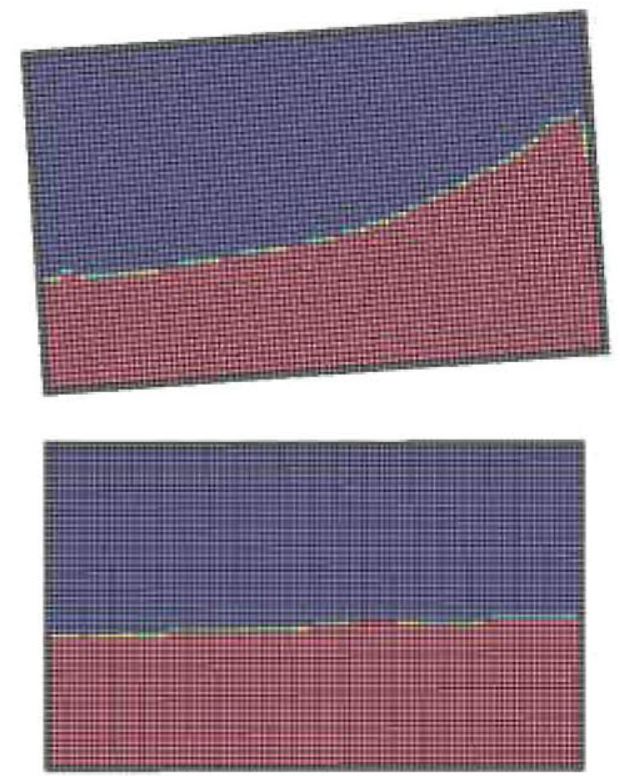

Figure 21-22. Kinematics capturing with LS-DYNA-max peak (upper) and min peak (lower).

Also in this case PRI and BV calculations are those which were run for a longer time, showing again a good stability, considering also the high nonlinearities and surface deformations involved in this case.

For what regards Sensor 3, different considerations have to be made. All methods are capable of capturing the sloshing impact on the tank top, however considering pressures, ranging the experimental value between 700 and $1000 \mathrm{~Pa}$ apart two initial higher peaks, all numerical methods present a rather variable behavior, with $\mathrm{BV}$ presenting oscillating values with higher peaks up to $1500 \mathrm{~Pa}$ and lower values with almost no impact, PRI and SPH with a similar trend and even higher peaks in some cases (2000 and $2500 \mathrm{~Pa}$, respectively) and UoS with less oscillating values with a decreasing tendency.

Moreover, both PRI and BV find a "quiet period" between $\mathrm{t}=15 \mathrm{~s}$ and $\mathrm{t}=20 \mathrm{~s}$ in which pressure peaks are very low; comparing it with the experiments, pressure peaks are effectively lower in that time range, even if not as low as obtained by calculations. SPH results are not available in correspondence to those times, and UoS seems to capture the pressure decrease even if data are not available to evaluate the possible pressure rises after $\mathrm{t}=17 \mathrm{~s}$.

It is worth underlining that experimental results in this case should be considered only as a signal of impact, while the absolute values can be affected by some problem because of very high frequency of impacts. 
Case $B$ - $T=T_{0}$ - Sensor 6

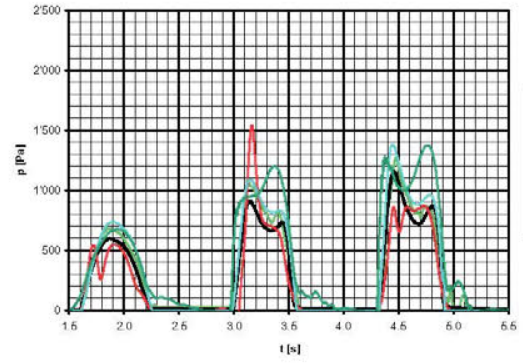

Case $B \cdot T=T_{n}$ - Sensor 6

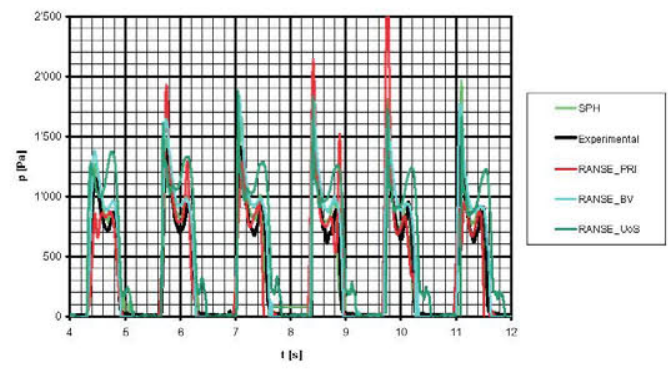

Figure 23. Test Case 3 Level $\mathrm{B}-\mathrm{T}=\mathrm{T}_{0}-$ Sensor 6 pressure time history-first oscillations (upper) and t $=4-12 \mathrm{~s}$ (lower).

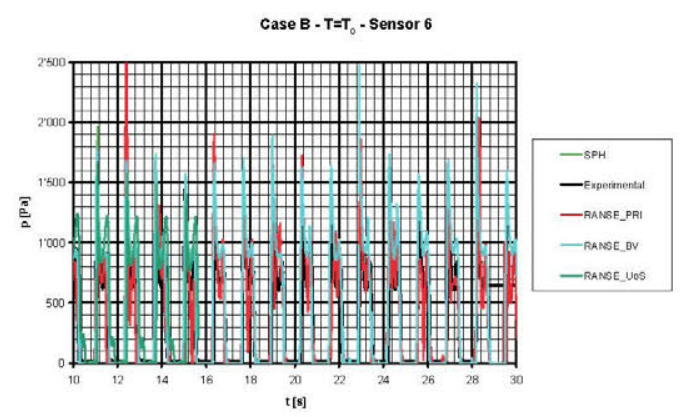

Figure 24. Test Case 3-Level $\mathrm{B}-\mathrm{T}=\mathrm{T}_{0}$ - Sensor 6 pressure time history for $\mathrm{t}=10-30 \mathrm{~s}$.

In following Fig. 26, results in terms of global torque, as evaluated with SPH and LS-DYNA are reported.

Global torque calculation with SPH in this case seem to be in a good agreement with experimental results, with a similar harmonic behavior and higher superimposed peaks (about 35\%), while RANS method by PRI shows a general overestimation of peaks, together with higher oscillations.
Case B - T $=\mathrm{T}_{0}$ - Sensor 3

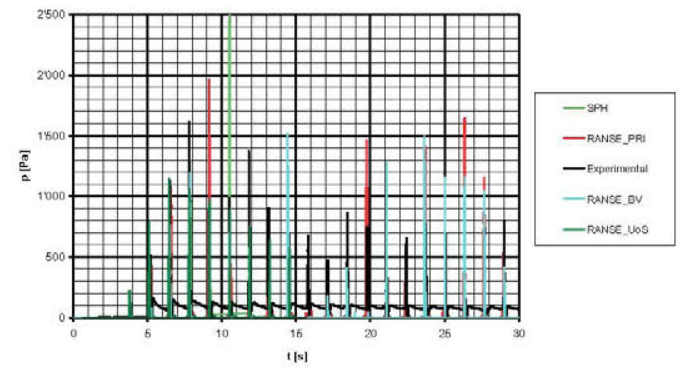

Figure 25. Test Case 3-Level $\mathrm{B}-\mathrm{T}=\mathrm{T}_{0}$ - Sensor 3 pressure time history up to $30 \mathrm{~s}$.

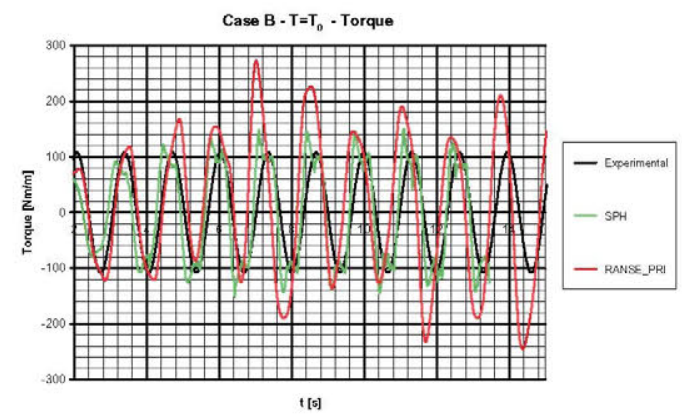

Figure 26. Test Case 2-Level B-T $=\mathrm{T}_{0}-$ Global torque time history up to $\mathrm{t}=15 \mathrm{~s}$.
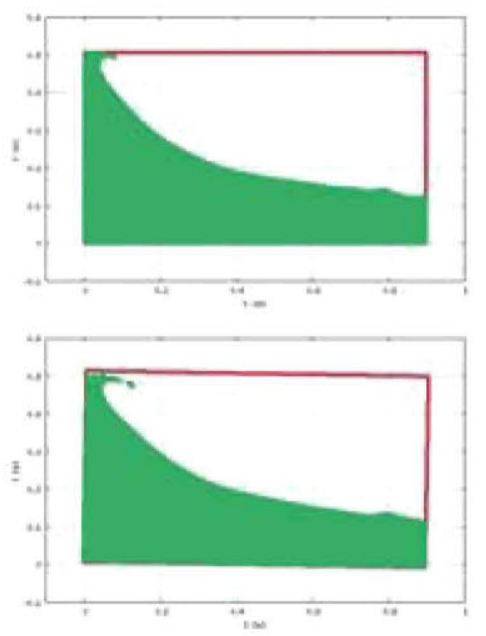

Figure 27-28. Kinematics capturing with $\mathrm{SPH}$-impact on tank top. 


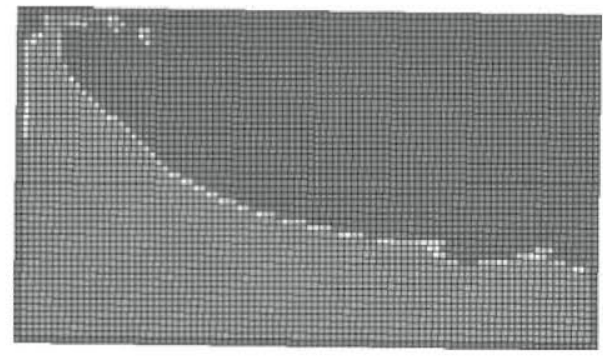

Figure 29. Kinematics capturing with LS-DYNA - impact on tank top.
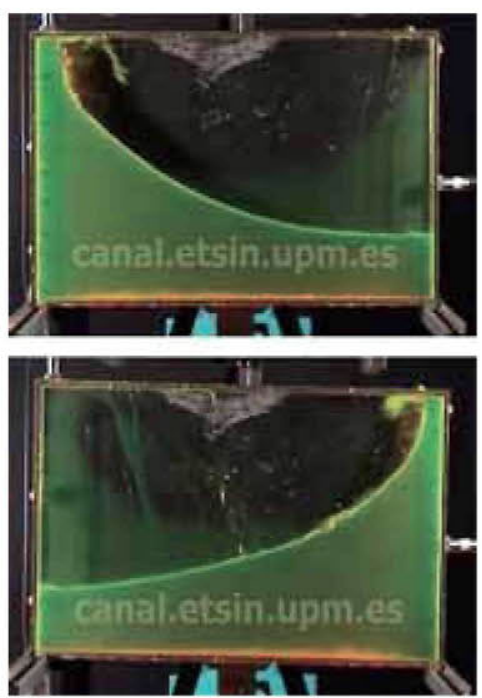

Figure 30-31. Kinematics during experiments - sloshing impacts at the two sides.

In Figs. 27-31, some examples of kinematics capturing with different methods adopted and experimental results are reported, showing the capability of each code to capture the impact on the tank top.

Calculations with SPH and LS-DYNA are in rather good agreement with each other from this point of view, and are able to capture the most significant phenomenon of sloshing impact on the tank top.

\section{CONCLUSIONS}

In present paper, an analysis of different numerical techniques for the evaluation of sloshing was carried out, comparing simulations with experimental results provided by CEHINAV-ETSIN-UPM for three significantly different test cases. In particular, attention was concentrated on pressures predicted by means of $\mathrm{SPH}$, an own developed RANS code and commercial software LS-DYNA and FLOW-3D; moreover, comparisons were also made to assess global torque calculation and kinematics capturing capability of some of the codes adopted.

From the analysis of results obtained, it can be concluded that:

- pressures predicted by the various numerical methods are rather satisfactory in general, with a sufficient correspondence with experiments, even if there are differences between each other (with pressures overestimation in some cases), with different capacity of different codes to capture correctly the sloshing phenomenon;

- the most problematic cases proved to be, as expected, the two involving large free surface deformations and violent impact phenomena, i.e. case 1 and 3 with roll period set equal to natural oscillation period of fluid; for those cases, most difficult calculations are those carried for sensors which are out of water for most the time;

- also for the less challenging case 2, some methods tend to overestimate peak pressures;

- application of commercial CFD codes, such as Flow-3D and LS-DYNA, is the most successful; codes provide satisfactory results and present an intrinsic robustness (especially the one adopted by $\mathrm{BV}$, while PRI results show anomalous higher oscillations) which own developed codes still have to reach, together with a comparably lower computational and/or setup time;

- SPH technique seems promising, and a satisfactory setting of parameters (which is one of the main shortcomings of the method) was achieved and utilized for all tests; moreover, pressure values are captured correctly without presence of strongly oscillating time histories, which is another important shortcoming of SPH; with these two achievements, results are comparable or even better in some cases than those obtained with commercial codes; however, the long computational time (about 10 and 40 hours for each computed second for case $\mathrm{A}$ and $\mathrm{B}$ respectively with respect to about $1.5-2 \mathrm{~h}$ with $\mathrm{UoS}$ method for case B and about $1.4 \mathrm{~h}$ for LS-DYNA, all on a conventional CPU), still prevents a systematic use of this technique, and requires further research efforts to consider it as applicable as the more usual RANS codes;

also own developed RANS code adopted by UoS seems promising, even if it still has to be further analyzed and calibrated, especially for what regards free surface treatment (and resulting pressures in its proximity) and time step/grid density adopted, with the aim of improving results already obtained, especially for test cases 2 and 3 . 
regarding global torque, results obtained with $\mathrm{SPH}$ and PRI RANS methods are in rather good agreement with each other and with the first harmonics values provided by UPM group, even if PRI values show higher oscillations and a tendency to overestimate peaks, while SPH produces a better correspondence.

Possible future research issues will be a further development of the own-made software, with the aim of getting a better insight into some problems still existing, like the long computational time with SPH (analyzing possible particles reductions without loosing accuracy and, in general, other different strategies to accelerate calculations) and the free surface treatment with the RANS code.

Moreover, possible future work may be related to the analysis of codes capability of capturing influence of air, fluid-structure interactions and possible different tank shapes (e.g. with baffles introduction).

\section{ACKNOWLEDGEMENTS}

The work was performed in the scope of the project MARSTRUCT, Network of Excellence on Marine Structures (<http://webmail.mar.ist.utl.pt/exchweb/ bin/redir.asp?URL=http://www.mar.ist.utl.pt/marstruct/>http://www.mar.ist.utl.pt/marstruct/), which has been financed by the EU through the GROWTH Programme under contract TNE3-CT-2003-506141.

Experimental data for the test cases were obtained in the framework of the project STRUCT-LNG (file numberCIT-370300-2005-16) leaded by the Technical University of Madrid (UPM) and funded by the Spanish Ministerio de Educacion y Ciencia in the program PROFIT 2005.

Messrs Chen and Temarel would like to acknowledge the support from Lloyd's Register Educational Trust, University of Southampton, University Technology Centre in Hydrodynamics, Hydroelasticity and Mechanics of Composites.

\section{REFERENCES}

Aliabadi, S., Johnson, A. \& Abedi, J., 2003. Comparison of finite element and pendulum models for simulation of sloshing. Computational Fluids 32, 535-545.

Aquelet, N., Souli, M., Gabrys, J. \& Olovson, L., 2003. A new ALE formulation for sloshing analysis. Structural Engineering and Mechanics 16 (4), 423-440.

Batchelor, G.K., 1967. An introduction to fluid mechanics. Cambridge Press.

Celebi, M.S. \& Akyildiz, H., 2002. Nonlinear modeling of liquid sloshing in a moving rectangular tanks. Ocean Engineering 29 (12), 1527-1553.
Chen, Y.G., Djidjeli, K., \& Price W.G., 2008. Numerical Simulation of Liquid Sloshing Phenomena in Partially Filled Containers. Computers \& Fluids, in press, available online.

Colagrossi, A. \& Landrini, M., 2003. Numerical simulation of interfacial flows by smoothed particle hydrodynamics, Journal of Computational Physics 191: 448-475.

Delorme, L. \& Souto Iglesias, A., 2007. Impact Pressure Test Case Description, ETSIN Report.

Delorme, L. \& Souto Iglesias, A., 2008. Impact Pressure Test Case Description, updated version with global moment calculations, ETSIN Report.

Delorme, L., Colagrossi, A., Souto-Iglesias, A., ZamoraRodriguez, R. \& Botia-Vera, E., 2008. A set of canonical problems in sloshing, Part I: Pressure field in forced roll - comparison between experimental results and $\mathrm{SPH}$, Ocean Engineering, In Press, Corrected Proof, Available online 17 October 2008, DOI: $10.1016 /$ j.oceaneng. 2008.09 .014 .

Faltinsen, O.M., Rognebakke, O.F. \& Timokha, A.N., 2005. Resonant three-dimensional nonlinear sloshing in a square-base basin. Part 2. Effect of higher modes. Journal on Fluid Mechanics 523, 199-218.

Frandsen, J.B., 2004. Sloshing motions in excited tanks. Journal of Computational Physics 196 (1), 53-87.

Gingold, R.A. \& Monaghan, J.J., 1977. Smoothed particle hydrodynamics: theory and application to non-spherical stars, Royal Astronautical Society 181: 375-389.

Graham, E.W. \& Rodriguez, A.M., 1952. The characteristics of fuel motion which affects airplane dynamics. Journal of Applied Mechanics 19, 381-388.

Lee, T., Zhou, Z. \& Cao, Y., 2002. Numerical simulations of hydraulic jumps in water sloshing and water impacting. Journal of Fluid Engineering 124, 215-226.

Lewison, G.R.G., 1976. Optimum design of passive roll stabiliser tanks. RINA Transactions and Annual Report, pp. $31-45$

Liu, G.R. \& Liu, M.B., 2003. Smoothed Particle Hydrodynamics-A Meshfree Particle Method, World Scientific Publishing.

Lloyd, A.R.J.M., 1989. Sea Keeping - Ship Behavior in Rough Weather, Ellis Horwood Limited, Chichester.

Monaghan, J.J., 1992. Smoothed Particle Hydrodynamics, Annual Rev. Astron. Astrophysics 30: 543-574.

Monaghan, J.J., 1994. Simulating free surface flows with SPH, Journal of Computational Physics 110: 399-406.

Oger, G., Doring, M., Alessandrini, B. \& Ferrant P., 2006. Two-dimensional SPH simulations of wedge water entries" Journal of Computational Physics 213: 803-822.

Sames, P.C., Marcouly, D. \& Schellin, T.E., 2002. Sloshing in rectangular and cylindrical tanks. Journal on Ship Research 46 (3), 186-200.

Souto-Iglesias, A., Delorme, L., Perez-Rojas, L. \& AbrilPerez, S., 2006. Liquid moment amplitude assessment in sloshing type problems with smooth particle hydrodynamics, Ocean Engineering Volume 33.

Stoker, J.J., 1957. Water waves. Pure and Applied Mathematics, vol. IV. Interscience, New York.

Schellin, T.E., Peric, M., El Moctar, O., Kim, Y.S. \& Zorn, T., 2007. Simulation of Sloshing in LNG Tanks, OMAE 2007, 26th International Conference on Offshore Mechanics and Arctic Engineering, San Diego, USA, 10-15 June 2007 
Temarel, P. (editor)., 2005. Slamming and green water scenarios and a comparison of theoretical slamming and green water impact models with model test results, MARSTRUCT Report MAR-D1-3-UoS-01 (2).

Temarel, P. \& Xu, L. (editors), 2007. Slamming scenarios and a comparison of theoretical slamming impact models with model test results, MARSTRUCT Report MAR-D13-UoS-02 (1)

Tveitnes, T., Ostvold, T.K., Pastoor, L.W. \& Sele, H.O., 2004. A sloshing design load procedure for membrane LNG tankers. Proceedings of the Ninth Symposium on Practical Design of Ships and Other Floating Structures, Luebeck-Travemuende, Germany.

Verhagen, J.H.G. \& Van Wijngaarden, L., 1965. Non-linear oscillations of fluid in a container. Journal on Fluid Mechanics 22 (4), 737-751.
Viviani, M., Savio, L., \& Brizzolara, S., 2007. Evaluation of Slamming Loads on Ship Bow Section Adopting SPH and RANSE Method, 32nd Congress of IAHR, International Association of Hydraulic Engineering and Research, Venice.

Viviani, M., Brizzolara, S., \& Savio, L. 2007. Evaluation of Slamming Loads on a Wedge-Shaped Section at Different Heel Angles adopting SPH and RANSE Methods, Conference of the International Maritime Association of the Mediterranean (IMAM 2007), Varna, ISBN 978-0-415-45521-3.

Viviani, M., Brizzolara, S. \& Savio, L., 2008. Evaluation of Slamming Loads using SPH and RANS Methods, Journal of Engineering for the Maritime Environment, in press. 